\title{
IMPACTOS DA DOENÇA DE PARKINSON NA VIDA DOS IDOSOS
}

Impacts of Parkinson's disease for olde age

Impactos de la enfermedad de Parkinson en la vida de los anciano

Joice Amorim Santos ${ }^{1}$, Everson Mateus Almeida Magalhães ${ }^{2}$, Isabely Fróes Correia ${ }^{3}$, Samara Alves França ${ }^{4}$, Saionara Silva Brito ${ }^{5}$, Caio Venancio Duarte Carvalho ${ }^{6}$, Tatiane Dias Casimiro Valença ${ }^{7}$, Pollyanna Viana Lima ${ }^{8}$

${ }^{1}$ Enfermeira, Faculdade Independente do Nordeste - FAINOR. Vitória da Conquista/BA, Brasil.

${ }^{2}$ Enfermeiro, Faculdade Independente do Nordeste - FAINOR. Vitória da Conquista/BA, Brasil.

${ }^{3}$ Graduanda do curso de Enfermagem, Faculdade Independente do Nordeste - FAINOR. Vitória da Conquista/BA, Brasil..

${ }^{4}$ Enfermeira, Faculdade Independente do Nordeste - FAINOR. Vitória da Conquista/BA,

${ }^{5}$ Graduanda do curso de Enfermagem, Faculdade Independente do Nordeste - FAINOR. Vitória da Conquista/BA, Brasil.

${ }^{6}$ Engenheiro Elétrico, Faculdade Independente do Nordeste - FAINOR. Vitória da Conquista/BA. Especialista em Engenharia Elétrica, Brasil.

${ }^{7}$ Fisioterapeuta, Doutora em Memória Linguagem e Sociedade. Docente Adjunta da Universidade Estadual do Sudoeste da Bahia, Jequié/BA, Brasil.

${ }^{8}$ Enfermeira, Doutora em Memória Linguagem e Sociedade. Docente da Faculdade Independente do Nordeste FAINOR. Vitória da Conquista/BA, Brasil.

*Correspondência: Faculdade Independente do Nordeste - FAINOR, Avenida Deputado, Av. Luís Eduardo Magalhães, 1305, Vitória da Conquista - BA, Brasil, CEP 45055-030.e-mail polly_vl@yahoo.com.br

Artigo recebido em 24/04/2019 aprovado em 07/10/2019 publicado em 04/12/2019.

\section{RESUMO}

O envelhecimento populacional no Brasil vem crescendo a cada década e atrelado a esse crescimento demográfico foi observado o aumento da incidência das doenças neurodegenerativas, como a Doença de Parkinson que acomete, principalmente pessoas de mais idade. O objetivo do estudo foi analisar os impactos da Doença de Parkinson em idosos. Trata-se de estudo descritivo, exploratório, qualitativo realizado no município de Vitória da Conquista BA, com 12 idosos com Doença de Parkinson. Os instrumentos para coleta dos dados foram: Questionário Sociobiodemográfico e Econômico e a Entrevista semiestruturada. Os dados foram analisados aplicando-se a análise de Conteúdo Temática emergindo duas categorias temáticas: Percepção dos idosos sobre a Doença de Parkinson, e Impactos da Doença de Parkinson nas atividades da vida diária. A doença de Parkinson gera impactos na vida dos idosos que podem ser percebidos a partir de sentimentos de tristeza e preocupação, limitação funcional e resiliência.

Palavras-chave: Idosos; Parkinson; Impactos; Envelhecimento.

\section{ABSTRACT}

Population aging in Brazil has been increasing every decade and, coupled with this demographic growth, the incidence of neurodegenerative diseases such as Parkinson's disease (PD) has been increasing, especially among older. The aim of the study was to analyze the impacts of Parkinson's disease in the elderly. This is a descriptive, exploratory, qualitative study carried out in the city of Vitoria da Conquista - BA, with 12 elderly people with Parkinson's Disease. The instruments for data collection were: Socio-demographic and Economic Questionnaire and the semi-structured interview. The data were analyzed applying the Thematic Content analysis emerging two thematic categories: Perception of the elderly on Parkinson's Disease and Impacts of Parkinson's Disease in the 
activities of daily living. Parkinson's disease impacts the lives of the elderly that can be perceived from feelings of sadness and worry, functional limitation, and resilience.

Keywords: Elderly; Parkinson; Impacts; Aging.

\section{RESUMEN}

El envejecimiento poblacional en Brasil viene creciendo cada década y vinculado a ese crecimiento demográfico fue observado el aumento de la incidencia de las enfermedades neurodegenerativas, como la Enfermedad de Parkinson que afecta, principalmente personas de más edad.El objetivo del estudio fue analizar los impactos de la enfermedad de Parkinson en ancianos. Se trata de un estudio descriptivo, exploratorio, cualitativo realizado en el municipio de Vitória da Conquista - BA, con 12 ancianos con enfermedad de Parkinson. Los instrumentos para recolección de los datos fueron: Cuestionario Sociobiodemográfico y Económico y la Entrevista semiestructurada. Los datos fueron analizados aplicando el análisis de Contenido Temático emergiendo dos categorías temáticas: Percepción de los ancianos sobre la Enfermedad de Parkinson, e Impactos de la Enfermedad de Parkinson en las actividades de la vida diaria. La enfermedad de Parkinson genera impactos en la vida de los ancianos que pueden ser percibidos a partir de sentimientos de tristeza y preocupación, limitación funcional y resiliencia.Palabras clave: Ancianos; Parkinson; impactos; Envejecimiento.

\section{INTRODUÇÃO}

$\mathrm{O}$ envelhecimento populacional vem se expandindo no Brasil devido a vários fatores, como a redução das taxas de natalidade e mortalidade, aumento da expectativa de vida e investimentos no setor da saúde e tecnologia (MANSO; GALERA, 2015). Simultaneamente, ocorreu o crescimento da incidência das doenças neurodegenerativas, como a Doença de Parkinson (DP) que acomete principalmente pessoas idosas (GOMES et al., 2014).

Conforme dados da Organização Mundial de Saúde (OMS) a DP atinge pessoas das mais diversas classes econômicas e etnias, com prevalência mundial de 100 a 200 casos a cada 100.000 habitantes, sendo que a maioria dos indivíduos acometidos são pessoas idosas. No que se refere à realidade brasileira, estimativas relatam que existe em média 200 mil pessoas parkinsonianas no país (BRASIL, 2014).

A DP é uma doença neurodegenerativa do sistema nervoso central que se manifesta através de sintomas motores caracterizados por tremor de repouso, instabilidade postural, rigidez de articulações, bradcinesia e sintomas não motores, como diminuição do olfato, distúrbios do sono, depressão e entre outros sintomas (BRASIL, 2014).
É considerada a segunda doença neurodegenerativa de longa sobrevida com maior prevalência no mundo e também com maior gasto financeiro para o indivíduo acometido, sua família e o Estado. Além disso, grande parcela desses idosos é acometida pela doença ainda durante a fase produtiva, interferindo de forma negativa na sua qualidade de vida (BOVOLENTA; FELÍCIO, 2016).

Nesta perspectiva, tendo em vista que o número de idosos vem crescendo em todo o mundo e consequentemente a prevalência das doenças neurodegenerativas e incapacitantes é imprescindível conhecer as necessidades de saúde da população idosa, para que se possam buscar soluções e intervir sobre determinados agravos que essas doenças podem provocar.

Estudos relatam que são muitos os impactos que os idosos com DP enfrentam, especialmente os relacionados a questões motoras e emocionais, como interferência nas Atividades de Vida Diária (AVD), Atividades de Instrumentais de Vida Diária (AIVD) e depressão (FILIPPIN et al., 2014; QUINTELLA et al., 2013). No que se refere aos impactos da doença na família estão relacionados à sobrecarga de trabalho, questões financeiras e emocionais dos cuidadores 
familiares (FILIPPIN et al., 2014; QUINTELLA et al., 2013).

Neste contexto, esse estudo justifica-se devido à necessidade de averiguar os principais impactos da DP na vida do idoso diagnosticado com a doença, com o intuito de evidenciar questões que pouco tem sido explorado na literatura atual, o que possibilitará aos profissionais da saúde uma melhor compreensão à cerca da complexidade da doença por meio da percepção dos próprios idosos. Ademais, pode contribuir para o desenvolvimento de estratégias que reduzam os impactos da DP e possibilite uma melhor qualidade de vida para estas pessoas (FILIPPIN et al., 2014; QUINTELLA et al., 2013).

Diante do exposto este estudo teve por objetivo analisar os impactos da DP na vida do idoso portador dessa doença no município de Vitória da Conquista-BA.

\section{MATERIAIS E MÉTODOS}

Este estudo é de natureza descritiva, exploratória, com abordagem qualitativa, realizado através da técnica "Bola de Neve", também conhecida como Snowball. Caracteriza-se como uma forma de amostra não probabilística na qual a escolha é intencional ou por conveniência, considerando as características particulares do grupo em estudo. Esta técnica utiliza cadeias de referência, um tipo de rede, ou seja, foram localizados os participantes iniciais do estudo que tiveram o perfil proposto para a pesquisa, esses participantes iniciais foram indicando outros participantes de interesse para a pesquisa da sua própria rede social, e assim por diante, de modo que um participante foi indicando outro até atingir o objetivo proposto pela pesquisa.

A vantagem deste método utilizando cadeias de referência é a facilidade do idoso com DP conhecer outro indivíduo que compartilha do mesmo problema. Outra característica importante deste método é a diversidade das cadeias de referência, pois podem atingir indivíduos pertencentes as mais diversas classes sociais, econômicas e culturais (VINUTO, 2014; BALDIN; MUNHOZ, 2011).

O estudo foi realizado no município de Vitória da Conquista - BA, nos domicílios dos idosos portadores da DP. O quadro de amostragens atingiu um ponto de saturação quando não houve novas indicações ou os novos indicados não trouxeram informações relevantes para a pesquisa (VINUTO, 2014; BALDIN; MUNHOZ, 2011).

Participaram do estudo 12 idosos que contemplaram aos seguintes critérios de inclusão, a saber: pessoas com 60 anos ou mais, ambos os sexos, diagnosticados com DP, que aceitaram participar voluntariamente do estudo, que residiam na cidade de Vitória da Conquista - BA e possuíam o cognitivo preservado, sendo avaliado pelo Mini Exame do Estado Mental (MEEM) (FOSTEIN et al., 1975). O MEEM é um questionário validado internacionalmente, composto de duas partes que aborda as questões de orientação, memória e atenção, com pontuação máxima de 21 pontos, sendo que a outra parte aborda as habilidades específicas como nomear e compreender, com pontuação máxima de 9 pontos, chegando a um escore final de 30 pontos contendo, para tanto, um ponto de corte entre 23/24 pontos, onde esse valor é sugestivo a déficit cognitivo. Sendo assim, quanto maior a escore no final do questionário melhor será o desempenho cognitivo do indivíduo que participará do estudo (FOSTEIN et al., 1975). Salienta-se, que o MEEM foi utilizado neste estudo apenas como critério de inclusão, ou seja, para definir os idosos que possuíam 
o cognitivo preservado para responder o questionário, bem como a entrevista.

Para a coleta de dados foram utilizados dois instrumentos: o questionário Sociobiodemográfico e Econômico e um roteiro para entrevista semiestruturada, elaborados pelos próprios pesquisadores. O questionário Sociobiodemográfico e Econômico apresentou questões sobre: sexo, idade, estado civil, religião, número de filhos, escolaridade, com quem mora, renda individual, renda familiar, entre outras. A entrevista semiestruturada para os idosos continha questões que abordavam: diagnóstico da DP; dificuldades enfrentadas na realização de atividades do dia-a-dia e convívio familiar e social. A entrevista foi gravada com a anuência dos idosos para posteriormente os dados serem transcritos e analisados pelos pesquisadores.

A análise dos dados ocorreu por meio da técnica de Análise de Conteúdo de Laurence Bardin (2011). Existem diversos tipos de técnicas para o desenvolvimento da análise de conteúdo, sendo que nesta pesquisa optou-se pela temático-categorial. Esta funciona com o desmembramento do texto em categorias, sendo que o critério de categorização nesse caso é semântico, ou seja, valoriza o significado das palavras, frases, parágrafos, manifestados por meio da linguagem dos sujeitos (BARDIN, 2011).

Somente após a aprovação do Comitê de Ética em Pesquisa (CEP) da Faculdade Independente do Nordeste (FAINOR) foi iniciado o processo de coleta dos dados, sendo todos os participantes informados e esclarecidos sobre a natureza da pesquisa, bem como seus objetivos, métodos, benefícios previstos, potenciais riscos e o incômodo que esta podia lhes causar, na medida de sua compreensão e respeitados em suas singularidades. Os idosos que aceitaram participar do estudo assinaram um Termo de
Consentimento Livre e Esclarecido (TCLE), contendo duas vias, sendo que uma delas ficou com o participante da pesquisa e a outra com o pesquisador.

Ressalta-se que todos os aspectos éticos e legais da Resolução 466/12 do Ministério da Saúde (BRASIL, 2013) e da Resolução 510/2016 (Brasil, 2016) foram seguidos rigorosamente. O projeto foi encaminhado ao CEP/FAINOR com Certificado de Apresentação para Apreciação Ética número (CAAE) $n^{\circ} 79195017.6 .0000 .5578$ e aprovação $n^{\circ} 2.418 .868$.

\section{RESULTADOS E DISCUSSÃO}

A análise do questionário Sociobiodemográfico e Econômico permitiu a construção do perfil dos idosos com DP participantes do estudo, observando-se que a maioria $58,3 \%$ (7) era do sexo masculino, $50 \%$ (6) se encontravam na faixa etária entre 71 a 80 anos; 50\% (6) residiam com companheiro, 41,7\% (5) eram alfabetizados, 91,7\% (11) possuíam renda individual de um salário mínimo, $83,3 \%$ (10) possuíam renda familiar entre 1 e 5 salários mínimos, $66,7 \%$ (8) se dizem de religião católica e 50\% (6) são de cor branca.

Por meio da análise das narrativas dos idosos participantes do estudo foram construídas duas categorias temáticas que se subdividiram em cinco subcategorias apresentadas no quadro 1 .

Quadro 1 - Categorias temáticas do estudo, Vitória da Conquista-BA, 2018.

\begin{tabular}{|l|l|}
\hline Categorias & \multicolumn{1}{c|}{ Subcategorias } \\
\hline $\begin{array}{l}\text { 1 - Percepção dos } \\
\text { idosos sobre a Doença } \\
\text { de Parkinson }\end{array}$ & $\begin{array}{l}\text { 1.1 Tristeza e preocupação } \\
\text { 1.2 Aceitação e Resiliência }\end{array}$ \\
\hline $\begin{array}{l}\text { 2 } \text { - Impactos da } \\
\text { Doença de Parkinson } \\
\text { nas atividades da vida } \\
\text { diária dos idosos }\end{array}$ & $\begin{array}{l}\text { 2.1 Dificuldades na } \\
\text { realização nas atividades da } \\
\text { vida diária } \\
\text { 2.2 Realização das } \\
\text { atividades da vida diária } \\
\text { sem dificuldades }\end{array}$ \\
\hline
\end{tabular}




\section{Categoria 1 - Percepção dos idosos sobre a doença de Parkinson}

No que se refere à percepção dos idosos frente à DP foi possível observar nas narrativas que os idosos demonstraram sentimentos negativos quando da descoberta da doença, em contrapartida outros idosos relataram ter reagido normalmente ao diagnóstico e as limitações trazidas pela DP, demostrando aceitação e resiliência. As limitações funcionais e cognitivas também foram apontadas nas narrativas dos idosos quando perguntados a respeito da sua percepção da doença.

Assim, essa categoria foi subdividida em duas subcategorias que apresentam a várias percepções dos idosos sobre a presença da DP em sua vida.

\section{Subcategoria 1.1 - Tristeza e preocupação}

O diagnóstico da DP ocasiona um grande impacto na vida dos idosos, levando a diversos sentimentos em relação à doença, uma vez que cada indivíduo reage de forma diversificada em relação aos problemas de saúde. O enfretamento da doença não é fácil e, muitas vezes vêm acompanhadas de sentimentos negativos, como tristeza, medo, angústias, não aceitação da doença, decepção, medo da dependência, entre outros (PETERNELLA; MARCON, 2009). Esses sentimentos foram observados nas falas dos idosos:

$\mathrm{Eu}$ fiquei preocupado, porque eu falei se for para prejudicar a mim, meu trabalho... E prejudicou, fiquei muito preocupado, triste também (IP 2).

Quando eu cheguei em casa, que eu comecei a chorar, assim... desesperada (IP 6).
Em estudo realizado por Gomes et al., (2014) ficou constatado que os idosos parkinsonianos participantes também apresentaram sentimento de tristeza em relação ao diagnóstico da DP, principalmente devido as limitações funcionais impostas pela doença. Os resultados do estudo de Santos et al., (2009) também estão de acordo com o presente estudo, demonstrando que os idosos apresentam alterações emocionais em relação à doença, levando ao sentimento de tristeza, desânimo e em alguns casos até a depressão.

Conviver com uma doença progressiva e incapacitante como a DP é motivo de muita preocupação para boa parte dos idosos que precisam se adaptar a novas mudanças em suas atividades cotidianas, como trabalho, relacionamentos, atividades sociais, mobilidade física e independência. A pessoa com DP tem que a cada dia lidar com os sintomas e as limitações vivenciando uma luta constante para manter sua autonomia e independência (VALCARENGHI et al., 2018) .

A limitação funcional está relacionada ao comprometimento físico, mental, social e emocional, sendo associada ao maior comprometimento clínico da DP, pois causam grandes impactos nas atividades vida diária, acarretamento piora na qualidade de vida dos idosos (RODRIGUES et al., 2014).

A DP apesar de ser considerada como uma desordem das funções motoras, também se caracteriza por alteração na função cognitiva, sendo essa considerada uma complicação bastante comum. O déficit de cognição em indivíduos com DP é devido à deficiência da função executiva, apresentando falta de concentração, dificuldades de raciocinar, distúrbios na memória, entre outros, o que acarreta dificuldades na organização e planejamento das tarefas e no comportamento do dia a dia (LEMES et al., 2016). 
Neste estudo ficou evidente que as limitações funcionais e cognitivas é uma realidade para os entrevistados, como mostra as narrativas:

É difícil por que minha profissão ficou de lado e eu gosto muito de trabalhar, gosto muita da minha profissão de eletricista, eu acho linda (IP 4).

É triste, querer fazer as coisas e não poder (IP 1).

Resultado semelhante foi encontrado no estudo de Rodrigues et al., (2014) no qual ficou demonstrado que os idosos apresentam déficits cognitivos e menor independência funcional quando comparados com idosos saudáveis e que quanto maior for o comprometimento clínico da doença pior será o impacto nas atividades funcionais. Já o estudo de Pinheiro et al., (2014) evidenciou ligeira dependência no comprometimento funcional, podendo ter relação com o estadiamento da doença, sendo que quanto mais avançado estiverem os sintomas, menor será a capacidade funcional desses indivíduos.

Assim, idosos parkinsonianos devem se adaptar a um novo estilo de vida por conta das dificuldades impostas pela doença, uma vez que passam a ter prejuízos funcionais e em outras dimensões, e isso pode ser alcançado por meio da conscientização dos idosos a respeito de suas limitações e da necessidade de receberem cuidados com a evolução da doença (PINHEIRO et al., 2014; GOMES et al., 2014).

As alterações funcionais e cognitivas podem impactar de maneira expressiva na atividade laboral da pessoa com DP, principalmente quando diagnosticada ainda em uma fase produtiva da vida ocasionando saída do mercado de trabalho, aposentadoria, impacto na vida econômica do individuo e sua família, isolamento social e até depressão (ALVAREZ et al., 2017). O trabalho tem todo um simbolismo e um aspecto significativo para o indivíduo, pois é fonte de renda, sustento, papel social, relacionamentos interpessoais, a pessoa se sente ativa perante a sociedade gerando autoestima.

Foi possível observar no discurso do IP 2 que o diagnóstico da DP refletiu em preocupação em relação ao seu trabalho, uma vez que a doença, com a sua evolução pode gerar incapacidade que dificulta ou impede determinados movimentos comprometendo a prática laboral. Dependendo da atividade profissional realizada, os impactos podem chegar ao afastamento do trabalho como evidenciaram os resultados do estudo de Valcarenghi et al., (2018) em que os indivíduos com DP enfrentaram dificuldades para realizar suas atividades e a pouca produtividade no trabalho. Essa situação de limitação, da falta de obter o sustento familiar, de se encontrar dependente gera sentimentos de infelicidade, de preocupação e até de revolta, pois o trabalho para os indivíduos é uma forma de se sentirem pertencentes a sociedade e de grande relevância no que se refere aos relacionamentos interpessoais e de autoestima.

Nesse contexto, Antunes e Moré (2016) consideram que o afastamento do trabalho de indivíduos com DP pode envolver múltiplas questões relacionadas à perda de papéis sociais e de poder gerando impactos importantes na vida social desses indivíduos. Os participantes do estudo de Valcarenghi et al., (2018) destacaram que no trabalho eles próprios ou os colegas de trabalho perceberam dificuldades no desempenho das suas atividades laborais e constataram que a produtividade não era mais a mesma tendo que se afastar de seu emprego.

Sendo assim, não apenas as limitações físicas ou funcionais devem ser levadas em consideração no 
cuidado ao idoso com DP, mas as consequências emocionais que essas mudanças podem trazer, como sentimentos negativos, tristeza e a ansiedade, principalmente porque estes sentimentos são desencadeadores de outras patologias, como a depressão, (VALCARENGHI et al., 2018). No entanto, com o decorrer do tempo alguns idosos vão percebendo a necessidade de se adaptarem a tal situação.

\section{Subcategoria 1.2 - Aceitação e resiliência}

Em se tratando de enfermidade, a resiliência é considerada como a capacidade que as pessoas têm de enfrentar a doença de forma positiva, aceitando as limitações e buscando formas de se adaptar a tal situação. A resiliência ainda permite que as pessoas tenham controle sobre os impactos negativos trazidos pela doença no que se refere às questões físicas, sociais, econômicas e emocionais (BOELL et al., 2016).

Os idosos do presente estudo relataram que ao serem diagnosticados com a DP precisaram aceitar e buscar conviver da melhor maneira, pois a partir daquele momento se iniciava um processo de modificações na sua vida, visto que a doença vai progredindo ao longo do tempo e suas consequências vão gerando cada vez mais limitações em seu viver. Sendo assim, é importante que o idoso seja estimulado pela família, pela sociedade, pelos profissionais que o assiste e por ele mesmo a assumir formas de enfrentamento da doença, de modo que a doença e suas consequências não venham a influenciar de forma negativa na qualidade de vida desses indivíduos (SOUZA et al., 2014).

Foi possível perceber no presente estudo que a maioria dos idosos parkinsonianos demonstraram aceitação e resiliência frente à DP, conforme evidenciaram as falas:

A gente acostuma, com o costume tudo fica normal. Tem 20 e tantos anos que convivo com ela, então já acostumei (IP 10).

Tem que acostumar com a doença, a gente estando doente tem que conformar, entregar para Deus e pedir para ele, para ele resolver minha vida, que só ele pode resolver (IP 6).

A respeito da população idosa é necessário que os mesmos sejam capazes e estejam preparados para encarar as dificuldades desta fase da vida e isso requer uma capacidade de resiliência, como descreve o estudo de Silva et al., (2012). E quando esse idoso é acometido por uma doença neurológica e crônica os sentimentos de aceitação e resiliência devem ser ainda mais aprimorados buscando um enfrentamento das dificuldades impostas pela doença e buscando uma melhor qualidade de vida.

O estudo de Lima et al., (2016) demonstrou que mesmo diante de uma doença crônica degenerativa que leva à diversas limitações os idosos demonstraram aceitação e sentimento de resiliência. Além disso, o mesmo estudo mostrou que os idosos sabem lidar com as adversidades da vida e busca superar tal situação para viver da melhor maneira possível de acordo com as limitações impostas pela doença (LIMA et al., 2016).

Com o uso da medicação também vai ocorrendo a diminuição dos sinais e sintomas da doença o que favorece a percepção positiva em relação ao enfrentamento DP pelo indivíduo (VALCARENGHI et al., 2018). A adoção de um plano de tratamento medicamentoso e fisioterapêutico são fundamentais para a pessoa com DP superar as consequências físicas e cognitivas da doença. Assim, a resiliência se apresenta como uma ferramenta de 
enfrentamento da doença crônica promovendo a aceitação e adoção de novos hábitos de vida (BOELL et al., 2016).

\section{Categoria 2 - Impactos da Doença de Parkinson nas atividades da vida diária dos idosos}

No presente estudo, alguns idosos referiram dificuldades para a realização das AVDs surgindo assim a subcategoria "Dificuldades na realização nas atividades da vida diária". Por outro lado, outros idosos relatam que a DP não tem influenciado no seu cotidiano, o que fez emergir a subcategoria "Realização das atividades da vida sem dificuldades".

\section{Subcategoria 2.1 - Dificuldades na realização}

\section{nas atividades da vida diária}

O idoso quando acometido por uma doença neurodegenerativa como a DP apresentam mudanças de ordem física que leva ao comprometimento na realização Atividades da Vida Diária (AVDs) que são atividades como vestir, banhar, alimentar, que até então eram executadas com independência passando a depender de outra pessoa para serem executadas sendo que com o decorrer do tempo e da progressão da doença essa dependência pode ser ainda maior (MUNIZ et al., 2016; GOMES et al., 2014; PINHEIRO et al., 2014; SILVA et al., 2014).

Porém, há de salientar que a ocorrência do comprometimento das atividades cotidianas vai depender da fase da doença em que o indivíduo se encontra. Sendo assim, a capacidade funcional dos parkinsonianos é mais acometida nas fases intermediária e tardia da doença (VALCARENGHI et al., 2018).

Pesquisa realizada por Pinheiro et al., (2014) indicou que a maior parte dos idosos parkinsonianos apresentou leve dependência funcional nas AVDs, o que pode está relacionado ao estágio inicial da DP, pois com avançar da doença capacidade funcional tende a diminuir e aumentar a dependência em relação aos cuidados de outras pessoas, o que gera impactos negativos na qualidade de vida.

No presente estudo, esta realidade também foi evidenciada, conforme pode ser vista nos trechos das narrativas:

Eu fiquei tentando fazer as coisas que eu fazia, pensando que eu ainda era aquela pessoa como era antes. Eu não parava, eu não gostava de ficar parado, sempre saia para qualquer serviço, para qualquer trabalho. Demorou um pouquinho para eu conformar que eu não sou mais aquela pessoa (IP 9).

Não consigo fazer mais nada, não dá para fazer as coisas dentro de casa, eu fazia muito minhas coisas, gostava eu mesmo fazer minhas coisas (IP 8).

Os trechos das falas evidenciaram que os idosos percebem as suas limitações nas AVDs e que a modificação no cotidiano surgiu após o desenvolvimento e progressão da DP. Os estudos de Gomes et al., (2014) e Valcarenghi et al., (2018) corroboraram com o presente estudo, os quais mostraram que as limitações impostas pela doença interferem consideravelmente nas atividades funcionais dos idosos parkinsonianos.

Conviver com doenças crônico-degenerativas como a DP requer uma compreensão dos idosos e seus familiares, pois devido às limitações impostas pela doença, o cotidiano dos idosos parkinsonianos tende a ficar comprometido já que este processo exige complexas adaptações na rotina da vida dos idosos (GOMES et al., 2014). 


\section{Subcategoria 2.2 - realização das atividades da} vida diária e instrumentais sem dificuldades

Nas narrativas de alguns participantes deste estudo ficou evidente que mesmo com a presença com uma doença neurodegenerativa incapacitante os idosos ainda conseguem realizar diversas atividades, conforme pode ser observada nas narrativas:

Continuo fazendo as coisas, faço comida, varro casa, jogo roupa na máquina (IP 12).

Minhas atividades continuam normais! Eu faço tudo sozinho, vou no banco sozinho, vou na roça sozinho, eu dirijo, faço tudo por minha conta, até agora não preciso de ajuda de ninguém (IP 10).

De acordo com o estudo de Silva (2015), os pacientes com DP apresentaram independência na realização das AVDs. Já o estudo de Pinheiro (2014) mostrou que os idosos parkinsonianos apresentaram pequena dependência funcional no que se refere às AVDs. Acredita-se que as diferenças encontradas nos dois estudos mencionados, assim como neste estudo, se referem ao estágio em que a doença de encontra em seu processo de desenvolvimento. A fase de evolução da doença é um fator importante na qualidade e na capacidade de realização das AVDs, bem como na percepção dos indivíduos frente à doença (RODRIGUES et al., 2014; FILIPPIN et al., 2014). Assim os idosos devem se preparar buscando maneiras de enfrentamento das limitações impostas pela doença desde o seu diagnóstico buscando tratamento físico e psicológico buscando conviver melhor com a doença (VALCARENGHI et al., 2018).

\section{CONCLUSÃO}

Com base nos achados do presente estudo conclui-se que a DP traz impactos na vida dos idosos e isso foi possível ser compreendido através das suas próprias narrativas. Foram evidenciados sentimentos de tristeza e preocupação dos idosos ao serem diagnosticados com uma doença neurodegenerativa, crônica, progressiva e incapacitante. As incertezas em relação ao trabalho, o sustento a independência impactaram na sua condição emocional e social.

Quanto às limitações funcionais e cognitivas impostas pela DP foi possível perceber que os idosos enfrentam cada um a sua maneira. Para alguns a doença é um fardo pesado que tem consequências danosas à sua qualidade de vida. Em contrapartida, alguns idosos mostraram aceitação e resiliência frente à doença. Além disso, ficaram evidentes os impactos da DP nas atividades de vida diária levando o idoso a dependência, necessitando sempre da ajuda de outra pessoa na realização de tarefas cotidianas. Entretanto, a maioria dos idosos ainda consegue realizar suas atividades básicas do dia-dia e até atividades mais complexas.

Nesse contexto, é necessário que os profissionais da área da saúde que prestam assistência à pessoa idosa com DP tenham um olhar mais atencioso e amplo aos vários aspectos que podem ser afetados com a doença, como a função, cognição, sentimentos e a forma de aceitação e enfrentamento da doença. É necessário desenvolver ações em educação saúde, orientações e ações de cuidado com os idosos com DP e seus familiares, a fim de minimizar os impactos produzidos pela doença e garantir uma melhor qualidade de vida para o idoso.

As limitações desse estudo foram principalmente às dificuldades para agendar visita nos domicílios dos idosos, já que não possui nenhum grupo de apoio no município que facilitasse o contato 
e a escassez de publicações científicas relacionadas à temática investigada para se construir um melhor embasamento teórico e uma comparação dos

Todos os autores declararam não haver qualquer potencial conflito de interesses referente a este artigo.

\section{REFERÊNCIAS}

ALVAREZ A.M.; VALCARENGHI R.V.; NUNES S.F.L.; SIEWERT J.S.; BAPTISTA R. The retirement impact in people with Parkinson disease during active age. Rev. Eletr. Enf., v.19, p. 19:a16,2017. Recuperado em 10 dez, 2018, de: https://revistas.ufg.br/fen/article/view/39607/23241

ANTUNES M.H.; MORÉ C.L.O.O. Aposentadoria, saúde do idoso e saúde do trabalhador: revisão integrativa da produção brasileira, Revista Psicologia Organizações e Trabalho, v.16, n.3, p.248-258, 2016. Recuperado em $10 \mathrm{dez}, 2018$, de: http://pepsic.bvsalud.org/pdf/rpot/v16n3/v16n3a04.pd $\underline{\mathrm{f}}$

BALDIN, N.; MUNHOZ, E. M. B. (2011). Snowball (Bola de neve): Uma técnica metodológica para pesquisa em educação ambiental comunitária. In: Congresso de Educação, 10. Seminário de Representações Sociais, Subjetividade e Educação SIRSSE, 1. Anais. Pontifícia Universidade Católica do Paraná, Curitiba. p. 1-13. Recuperado em $10 \mathrm{dez}$, 2018, de:

http://educere.bruc.com.br/CD2011/pdf/4398 2342.p df

BARDIN, L. (2011). Análise de conteúdo. Lisboa: Edições 70.

BOELL, J. E. W.; SILVA, D. M. G. V.;

HEGADOREN, K. M. Fatores sociodemográficos e condicionantes de saúde associados à resiliência de pessoas com doenças crônicas: um estudo transversal. Rev. Latino-Am. Enfermagem, v.24, p.e2786, 2016. Recuperado em 10 dez, 2018, de: http://www.scielo.br/scielo.php?script=sci issuetoc\& pid=0104-116920160001\&lng =pt\&nrm=iso\&page $=3$

BOVOLENTA, T. M.; FELÍ́CIO, A. C. O doente de Parkinson no contexto das Políticas Públicas de Saúde no Brasil. Einstein, v.14, n.3, p.7-9, 2016. Recuperado em 10 dez, 2018, de: resultados encontrado nesse estudo com outras realidades estudadas.

https://journal.einstein.br/pt-br/article/o-doente-deparkinson-no-contexto-das-politicas-publicas-desaude-no-brasil/

BRASIL, Ministério da Saúde. Resolução 466, de 2012. 2013 Recuperado em $10 \mathrm{dez}$, 2018, de: http://conselho.saude.gov.br/resolucoes/2012/reso466 . $\mathrm{pdf}$

Conheça os sintomas do mal de Parkinson. Portal Brasil, 2014. Recuperado em $10 \mathrm{dez}, 2018$, de: http://www.brasil.gov.br/saude/2014/10/conheca-ossintomas-do-mal-de-parkinson.

Resolução no 510, de 07 de abril de 2016.

Recuperado em 17 dez, 2018, de:

http://www.fainor.com.br/v2/wp-

content/uploads/2016/01/Resolucao-CNS-510-2016-

Normas-aplicaveis-a-pesquisas-em-ciencias-

humanas-e-sociais.pdf.

FILIPPIN, N. T.; MARTINS, J. S.; LIBERA, L. B. D.; HALBERSTADT, B. F.; SEVERO, A. R.

Qualidade de vida de sujeitos com doença de

Parkinson e seus cuidadores. Fisioter. Mov., v. 27, n.1, p.57-66, 2014. Recuperado em $17 \mathrm{dez}, 2018$, de: http://www.scielo.br/pdf/fm/v27n1/0103-5150-fm27-01-0057.pdf

FOLSTEIN, M.; FOLSTEIN, S.; MCHUGH, P. "Mini-mental state". A practical method for grading the cognitive state of patients for the clinician. J Psychiatr Res, v.12, n.3, p.189-198, 1975.

Recuperado em 20 dez, 2018, de: https://www.sciencedirect.com/science/article/pii/002 $\underline{2395675900266}$

Gomes, A. R.; Daher, D. V. \& Fonseca, T. C. (2014). A interrupção do cotidiano imposta pela Doença de Parkinson: perspectivas de idosos parkinsonianos.

Rev de Enfermagem, UFPE, v.8, n.5, p.1296-301, 2014. Recuperado em $15 \mathrm{dez}, 2018$, de:

https://periodicos.ufpe.br/revistas/revistaenfermagem/ article/viewFile/9812/9987

LEMES, L. B.; BATISTETTI, C. L.; ALMEIDA, I. A.; BARBOZA, N. M.; TERRA, M. B.; BUENO, M. E. B.; SANTOS, S. M. S. Desempenho cognitivoperceptual de indivíduos com doença de Parkinson 
submetidos à fisioterapia. ConScientiae Saúde.,v.15, n.1, p.44-52. Recuperado em 20 dez, 2018, de:

https://www.redalyc.org/articulo.oa?id=92946649006

LIMA, P. V.; VALENÇA, T. D. C.; REIS, L. A.

Envelhecer com dependência funcional: Construindo estratégias de enfrentamento. Rev Pesq Saúde, v.17, n.2, p.96-101, 2016. Recuperado em 17 dez, 2018, de:

http://www.periodicoseletronicos.ufma.br/index.php/r evistahuufma/article/view/6082

MANSO, M. E. G.; GALERA, P. B. Perfil de um grupo de idosos participantes de um programa de prevenção de doenças crônicas. Estud. interdiscipl. envelhec., v.20, n.1, p.57-71, 2015. Recuperado em 17 dez, 2018, de: https://seer.ufrgs.br/RevEnvelhecer/article/view/4126 $\underline{4}$

MUNIZ, E. A.; AGUIAR, M. F. DOS S.; BRITO, M. DA C. C.; FREITAS, C. A. S. L.; MOREIRA, A. C. A.; ARAÚJO, C. R. C. Desempenho nas atividades básicas da vida diária de idosos em Atenção Domiciliar na Estratégia Saúde da Família. Revista Kairós Gerontologia, v. 19, n.2, p.133-146, 2016. Recuperado em 17 dez, 2018, de: https://revistas.pucsp.br/kairos/article/view/30365

PETERNELLA, F.M.N.; MARCON, S.S.

Descobrindo a Doença de Parkinson: impacto para o parkinsoniano e seu familiar. Rev Bras Enferm., v.62, n.1, p.25-31, 2009. Recuperado em $17 \mathrm{dez}$, 2018, de:

http://www.scielo.br/pdf/reben/v62n1/04.pdf.

PINHEIRO, I.M.; SANTOS, L.L.S.; PAULA, L.C.N.; COSTA, A.C.N. Impacto da Doença de Parkinson na funcionalidade e qualidade de vida de idosos em uma unidade de referência geriátrica na cidade de Salvador - Bahia. Rev. Ciências Médicas e Biológicas, v.13, n.3 (especial), p.292-297, 2014. Recuperado em 17 dez, 2018, de: https://portalseer.ufba.br/index.php/cmbio/article/vie w/12933

QUINTELLA, R.S.; SACHETTI, A.; WIBELINGER, L.M.; OLIVEIRA, S.G. (2013). Qualidade de vida e funcionalidade na doença de Parkinson. RBCEH, v.10, n.1, p.104-112.

Recuperado em 16 dez, 2018, de: http://www.scielo.br/pdf/fm/v27n1/0103-5150-fm27-01-0057.pdf

RODRIGUES, A.V.; LEMES, L.B.; RODRIGUES, A.S.; SOUZA, R.B.; SANTOS, S.M.S. Avaliação do Sistema Perceptual em Idosos Saudáveis e em Idosos com Doença de Parkinson. Rev Neurociências, v.22, n.2, p.189-194, 2014.

Recuperado em 17 dez, 2018, de: http://www.revistaneurociencias.com.br/edicoes/2014 2202/original/901original.pdf

SANTOS, I.S.C.; MENEZES, M.R.; SOUZA, A.S. Concepções de idosos sobre a vivência com a Doença de Parkinson. Rev. enferm. UERJ, v.17, n.1, p.6974, 2009. Recuperado em 17 dez, 2018, de: http://www.facenf.uerj.br/v17n1/v17n1a13.pdf.

SILVA, E.A.P. C.; SILVA, P.P.C.; MOURA, P.V.; SANTOS, A.R.M.; DABBICCO, P.; AZEVEDO, A.M.P.; FREITAS, C.M.S.M. Resiliência e saúde: uma análise da qualidade de vida em idosos.

ConScientiae Saúde, v.11, n.1, p.111-118, 2012.

Recuperado em 17 dez, 2018, de:

https://www.redalyc.org/html/929/92923617015/

SILVA, D.C.L.; VIANNA, E; MARTINS, C.P.; MARTINS, J.V.; RODRIGUES, E.C.; OLIVEIRA, L.A.S. Perfil dos indivíduos com doença de Parkinson atendidos no setor de fisioterapia de um hospital universitário no Rio de Janeiro. Revista Brasileira de Neurologia, v.51, n.4, p.126-140, 2015. Recuperado em $17 \mathrm{dez}, 2018$, de:

http://files.bvs.br/upload/S/01018469/2015/v51n4/a5407.pdf

SILVA, P.C.S.; FERNANDES, A.C.B. C.; TERRA, F.S. Avaliação da depressão e da capacidade funcional em idosos com Doença de Parkinson. Rev enferm UFPE, v.8, n.7, p.1920-1927, 2014.

Recuperado em $17 \mathrm{dez}, 2018$, de:

https://periodicos.ufpe.br/revistas/revistaenfermagem/ article/viewFile/23431/19122

SOUZA, J.M.; BARBOSA, A.C.; SILVA, A.L.F.; CAMPOS JÚNIOR, A. P. Doença de Parkinson: Atribuição de Enfermagem na interação FamíliaDoente. Rev eletrônica UNIVAR, v.1, n.11, p.96101, 2014. Recuperado em $17 \mathrm{dez}, 2018$, de: http://revista.univar.edu.br/index.php/interdisciplinar/ $\underline{\text { article/view/281 }}$

VALCARENGHI, R.V.; ALVAREZ, A.M.; SANTOS, S.S.C.; SIEWERT, J.S.; NUNES, S.F.L.; TOMASI, A.V.R. O cotidiano das pessoas com a doença de Parkinson. Rev Bras Enferm., v.71, n.2, p.293-300, 2018. Recuperado em $17 \mathrm{dez}, 2018$, de: http://www.scielo.br/scielo.php?script=sci_arttext\&pi $\mathrm{d}=\mathrm{S} 0034-$

$\underline{71672018000200272 \& \operatorname{lng}=p t \& n r m=i s o \& t \operatorname{lng}=p t}$

VINUTO, J. A amostragem em Bola de Neve na pesquisa qualitativa: um debate em aberto.

Temáticas, v.22, v.44, p. 203-220, 2014. Recuperado em $17 \mathrm{dez}, 2018$, de:

https://www.ifch.unicamp.br/ojs/index.php/tematicas/ article/view/2144/1637 